\title{
Stable CAAC-based Ruthenium Complexes for Dynamic Olefin Metathesis Under Mild Conditions
}

\author{
Oleksandr Kravchenko, ${ }^{\mathrm{a}}$ Brian J.J. Timmer, ${ }^{\mathrm{a}}$ Maurice Biedermann, ${ }^{\mathrm{a}}$ A. Ken Inge, ${ }^{\mathrm{b}}$ and \\ Olof Ramström*a,c,d
}

${ }^{a}$ Department of Chemistry, KTH - Royal Institute of Technology, Teknikringen 36, S-10044 Stockholm, Sweden; ${ }^{b}$ Stockholm University, Department of Materials and Environmental Chemistry, Svante Arrhenius väg 16C, S-10691 Stockholm, Sweden; ${ }^{c}$ Department of Chemistry, University of Massachusetts Lowell, One University Ave., Lowell, MA 01854, USA; Email: olof ramstrom@uml.edu; ${ }^{d}$ Department of Chemistry and Biomedical Sciences, Linnaeus University, SE-39182 Kalmar, Sweden

\begin{abstract}
A series of olefin metathesis catalysts bearing cyclic (alkyl)(amino)carbene (CAAC) ligands of varying size and steric demand has been synthesized and evaluated in ring-closing-, self-, and cross-metathesis reactions at room temperature. The catalysts were also probed for potential applications in dynamic covalent chemistry. The majority of the catalysts showed high stability, and remained active in the reaction mixtures for several days, including in methanol-based solutions. Higher temperatures could be used to control the reactivity towards sterically challenging substrates, enabling formation of tetrasubstituted olefins. The CAAC complexes exhibited remarkable functional group tolerance towards heteroaromatic and nucleophilic additives, making them potentially useful in the screening of biologically active compounds.
\end{abstract}

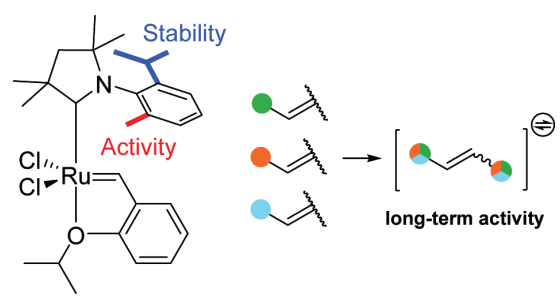

\section{INTRODUCTION}

Shortly after their discovery, ${ }^{[1,2]}$ cyclic (alkyl)(amino)carbenes (CAACs) have found numerous applications in transition metal- and main group chemistry. ${ }^{[3-5]}$ Being more $\sigma$-donating analogs of $\mathrm{N}$-heterocyclic carbenes (NHCs), CAACs can drastically change the electronic properties of metal-carbene complexes, ${ }^{[6]}$ for example resulting in catalysts that are particularly efficient in ethenolysis. ${ }^{[7-10]}$ Although the first CAAC-based catalysts did not exhibit improved activity in cross-metathesis processes compared to their NHC counterparts, recent reports have revealed that CAACs can enable reactivities that cannot be achieved with conventional catalysts. ${ }^{[10-12]}$ Stable bis-CAAC ruthenium complexes have thus become promising olefin metathesis catalysts, exhibiting high turnover numbers enabling new strategies in the synthesis of Hoveyda-Grubbs $2^{\text {nd }}$ generation catalysts. ${ }^{[13-15]}$

Many catalysts, including Grubbs $1^{\text {st }}$ generation (GI-Bn), Grubbs $2^{\text {nd }}$ generation $($ GII), Hoveyda-Grubbs $1^{\text {st }}$ generation $\left(\right.$ HGI), and Hoveyda-Grubbs $2^{\text {nd }}$ generation (HGII) catalysts, as well as CAAC complexes $\mathbf{1}$ and $\mathbf{3}$ (Figure 1 ), exhibit high kinetic E/Z-selectivity at low conversions, however reaching thermodynamic equilibria over time. ${ }^{[10]}$ This feature of the metathesis reaction has suggested the use of this transformation in dynamic covalent chemistry, ${ }^{[16-29]}$ although with severe limitations in substrate scope. ${ }^{[30-37]}$ As dynamic 
systems are often used for the discovery of bioactive compounds presenting a number of polar functional groups, the ability to equilibrate a mixture of olefins together with enhanced stability makes CAAC-based catalysts promising tools in dynamic chemistry.

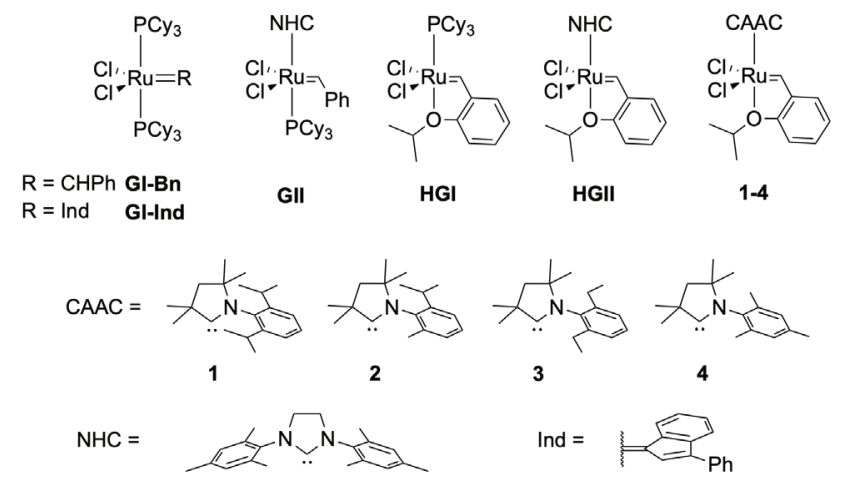

Figure 1: Commonly used NHC-based olefin metathesis catalysts (GI-Bn, GI-Ind, GII, HGI, HGII) and CAACbased complexes used in this study (1-4).

Applications of olefin metathesis in synthesis are sometimes limited due to poor functional group tolerance. ${ }^{[38]}$ Decomposition/poisoning of the catalyst are often important processes that determine the overall efficiency. ${ }^{[38-41]}$ On the other hand, the unique reactivity of CAAC-based catalysts is attributed to the increased stability of the reactive carbene and metallacyclobutane intermediates generated in situ. ${ }^{[9,15,42]}$ Therefore these catalysts can potentially be used under challenging conditions, such as in aqueous environment and with biologically relevant substrates. However, the complete substrate scope and functional group compatibility of CAAC-based catalysts have not been determined.

These challenges have been addressed in the present study, where we describe efficient synthesis protocols to a series of remarkably stable, yet active, CAAC-based olefin metathesis catalysts, and their evaluation in ring-closing-, self-, and cross-metathesis transformations. The functional group compatibility was furthermore evaluated, underscoring the high structural stabilities and showing that the CAAC complexes exhibited high tolerance towards heteroaromatic and nucleophilic additives. The complexes were also evaluated for use in dynamic covalent chemistry, where the results show that CAAC-based catalysts display high potential for generation and application of dynamic systems.

\section{RESULTS AND DISCUSSION}

\section{Catalyst synthesis}

The series of CAAC-based catalysts studied is displayed in Figure 1 (compounds 1-4). The structures possess small variations in the degree of steric bulk around the ruthenium core, thereby enabling detailed evaluation of the activities and stabilities. It was in this case expected that these variations would result in catalysts spanning the appropriate range of highly active to more stable structures, with the potential of identifying optimal complexes for different applications.

Two common synthetic routes towards NHC-based olefin metathesis catalysts start with a phosphino-ruthenium(II) precursor complex and an imidazolinium salt. ${ }^{[4]}$ The first route utilizes HGI as a starting material, and has been shown to be applicable also in the synthesis of CAAC-bearing complexes 1 and 3. ${ }^{[12]}$ However, this strategy could not be used to yield catalyst 4 , due to the shorter lifetime of the carbene. ${ }^{[9,12]}$ The other approach, based on ruthenium indenylidene complexes, was also unsuccessful in this case, and recent studies 
revealed that bis-CAAC adducts form instead. ${ }^{[15]}$ Further investigations of the activity of these complexes in olefin metathesis opened up a new strategy, which was successfully applied in the synthesis of a series of CAAC-based analogs of HGII. ${ }^{[15]}$ This approach enabled the expansion of the family of olefin metathesis catalysts to less bulky and potentially more reactive analogs.

Catalyst 1 was synthesized using the route based on HGI and the corresponding pyrrolidinium starting material in the presence of KHMDS in toluene. Catalysts $\mathbf{2 - 4}$, on the other hand, were synthesized following an approach utilizing isolated bis-CAAC complexes (Figure 2). In addition to obtaining catalysts $\mathbf{2}$ and $\mathbf{3}$, this protocol allowed the synthesis of catalyst 4, carrying a demanding mesityl substituent, which was previously reported impossible to synthesize. ${ }^{[12,14]}$ In contrast to the recently reported three-step-one-pot method, ${ }^{[15]}$ this procedure furthermore resulted in easier purification and generally higher yields $(30-90 \%)$.

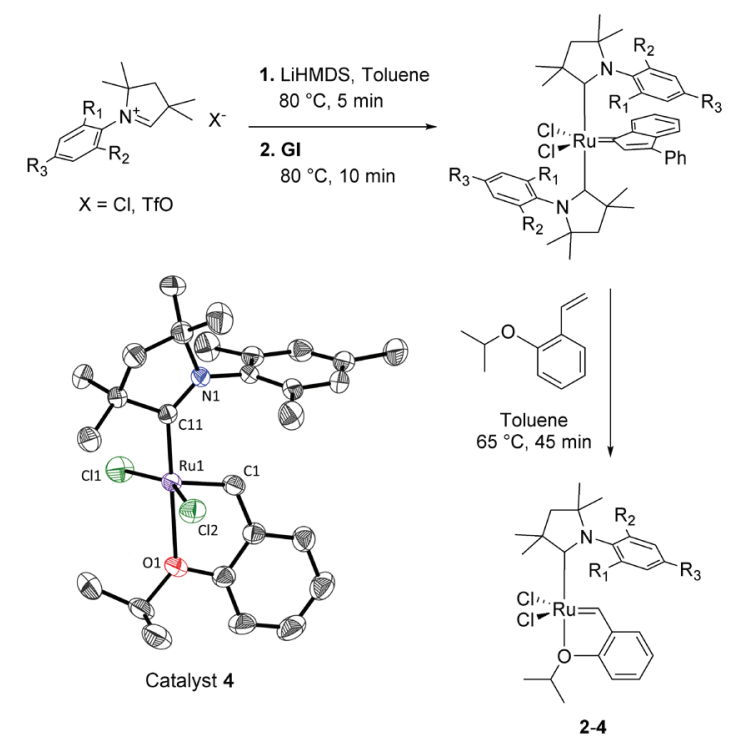

Figure 2: Synthesis of catalysts 2-4 via bis-CAAC complex; inset: X-ray crystal structure of catalyst 4 (thermal ellipsoids drawn at 50\% probability; hydrogen atoms omitted for clarity).

X-ray crystallographic analysis showed that catalyst $\mathbf{4}$ had the same conformation in the solid state as catalysts $\mathbf{1 - 3}$, with the $N$-aryl ring located above the benzylidene hydrogen (Figure 2). The $\mathrm{Ru}-\mathrm{C}_{11}(1.934 \AA)$ and $\mathrm{Ru}-\mathrm{O}(2.329 \AA)$ distances in catalyst 4 proved similar to those found in catalyst $\mathbf{1}\left(\mathrm{Ru}-\mathrm{C}_{11}: 1.930 \AA, \mathrm{Ru}-\mathrm{O}: 2.325 \AA\right)$, indicating low influence of the $N$-aryl aliphatic substituents on the $\sigma$-donating properties of the carbene. ${ }^{[12]}$ Therefore all differences in reactivity between catalysts $\mathbf{1 - 4}$ should be attributed to secondary steric factors of the complexes.

\section{Ring-closing metathesis}

All four complexes were tested in the formation of cyclic di-, tri- and tetrasubstituted olefins through ring-closing metathesis (RCM) of substrates 5-7 (Table 1 ). In complement to previous results with catalysts $\mathbf{1}$ and $\mathbf{3}^{[12]}$ cyclization of compounds 5 and $\mathbf{6}$ proceeded smoothly and were completed in 15 and $30 \mathrm{~min}$ at $40{ }^{\circ} \mathrm{C}$ with catalysts $\mathbf{2 , 3}$ and $\mathbf{4}$, respectively (Table $\underline{1}$, entries 2-3). Catalyst 1, however, did in this case not exhibit high activity in these cases, resulting in $95 \%$ conversion in $28 \mathrm{~h}$ and $172 \mathrm{~h}$, respectively. These results point to the importance of the benzylic methyl group(s) of the CAAC aryl moiety in retarding the RCM 
process, where replacement of a methyl group in benzylic positions 2 and/or 6 with a hydrogen significantly enhanced the reaction rates. The results also demonstrate the high stability of catalyst $\mathbf{1}$, being active for long periods of time at elevated temperature.

It has been shown that the activity of the catalysts can be significantly influenced by temperature. ${ }^{[12]}$ In particular, ring-closing metathesis of compounds 5 and $\mathbf{6}$ can be accomplished with catalyst 1 at $60{ }^{\circ} \mathrm{C}$ in 3.3 and $10 \mathrm{~h}$, respectively. To compare these results with catalysts 2-4, RCM of compound 5 was performed at room temperature, predictably resulting in longer reaction times. The observed catalyst activities appeared to be in accordance with the steric bulk of the $N$-aryl ring (Table 1 , entry 1 ), supporting the hypothesis that the catalyst activity is influenced by the steric interaction between the parallel alkylidene and $N$-aryl groups. ${ }^{[12,44]}$ Moreover, the replacement of one isopropyl group in catalyst $\mathbf{1}$ with a methyl moiety (catalyst 2) resulted in a dramatic increase in activity, however only slightly improved by replacement of the second isopropyl group (catalyst 4 ). The crucial difference between the $2,6{ }^{i}{ }^{i}{ }_{2}-$ and the $2{ }^{i} \operatorname{Pr}-6-\mathrm{Me}$ moieties can be explained by the selective rotation of the benzylidene ligand towards the less substituted side of the $\mathrm{N}$-aryl ring in catalyst 2.

Table 1: Activity of Ru-complexes 1-4 in RCM of selected substrates $(R=C O O E t) .{ }^{a}$

\begin{tabular}{|c|c|c|c|c|c|c|}
\hline Entry & Substrate & $\mathbf{T}\left({ }^{\circ} \mathbf{C}\right)$ & 1 & 2 & 3 & 4 \\
\hline 1 & & 22 & $372 \mathrm{~h}(95 \%)^{b}$ & $185 \min (95 \%)$ & $151 \min (95 \%)$ & $93 \min (95 \%)$ \\
\hline 2 & & 40 & 28 h $(95 \%)$ & $>15 \min (98 \%)$ & $>15 \min (98 \%)$ & $>15 \min (98 \%)$ \\
\hline 3 & & 40 & 172 h $(95 \%)$ & $30 \min (95 \%)$ & $30 \min (96 \%)$ & $30 \min (93 \%)$ \\
\hline 4 & & 60 & 30 h $(95 \%)$ & - & - & - \\
\hline 5 & 7 & 40 & $0 \%$ & $0 \%$ & $0 \%$ & $0 \%$ \\
\hline 6 & & 80 & $0 \%$ & 400 h $(5 \%)$ & 480 h $(22 \%)$ & $70 \mathrm{~h}(6 \%)$ \\
\hline
\end{tabular}

Although it was reported that catalysts $\mathbf{1}$ and $\mathbf{3}$ are not capable of forming tetrasubstituted olefins, ${ }^{[12,45]}$ prolonged reaction time along with higher temperatures $\left(>3 \mathrm{~d}\right.$ at $\left.80{ }^{\circ} \mathrm{C}\right)$ yielded the RCM product of compound 7 (Table 1, entry 6). Here, the difference in reactivity towards compounds 6 and 7 reflects the difference between the initiation rate of the allyl and methallyl moieties. Obviously, the less substituted allyl group is considerably more reactive in metathesis reactions, and, thus, both compounds $\mathbf{5}$ and $\mathbf{6}$ enter the catalytic cycle quickly and cyclize in short times.

\section{Self-metathesis}

The ability of the catalysts to accelerate self-metathesis processes was next explored, where allyl alcohol was first selected as a model substrate owing to its superior performance in these transformations. ${ }^{[46,47]}$ The metathesis process of allyl alcohols is believed to occur through hydrogen bonding, ${ }^{[48-50]}$ and, to some extent, coordination to the ruthenium center. ${ }^{[48]}$ In order to assess these types of effects in the reactions with the CAAC-based catalysts, allyl acetate (lacking hydrogen bond donor) and allyl benzene (free of any specific interactions) were added to the substrate pool.

Surprisingly, all catalysts exhibited low reactivities towards allyl alcohol, resulting in only $10-35 \%$ conversion (Figure S1). In most cases, the reaction progress slowed down significantly within a short period of time, with complete disappearance of the catalyst signal in the NMR spectrum. However, after a small decrease, the conversion started growing again slowly. These results suggest early catalyst decomposition followed by slow catalysis by 
unidentified $\mathrm{Ru}$ species formed during decomposition. Nevertheless, it is not clear why such an unusual behavior is triggered by allyl alcohol. Similarly, poor efficiency towards allyl acetate was observed, affording only slightly improved conversion in average (Figure S2), indicating the low influence from $\mathrm{H}$-bond donation in this case.

On the other hand, the self-metathesis process of allyl benzene reached about $50 \%$ conversion at room temperature with catalysts 1-3 (Figure $\underline{3}$, Figure S4), suggesting this to be the equilibrium position for this substrate, independent from catalyst loading (Figure S5). The lifetime of catalyst $\mathbf{4}$ appeared in this case to be shorter than the time required to establish the equilibrium between all metathesis products. No isomerization to the more stable $\beta$-methylstyrene or formation of other side-products occurred. However, the kinetic profile of this reaction suggests a two-step process with a slow initiation step, also observed for some NHC-based catalysts. ${ }^{[51]}$ The long induction time of catalyst $\mathbf{1}$ is in accordance with the RCM results and supports the hypothesis of the determining role of the ortho-substitution pattern for initiation.

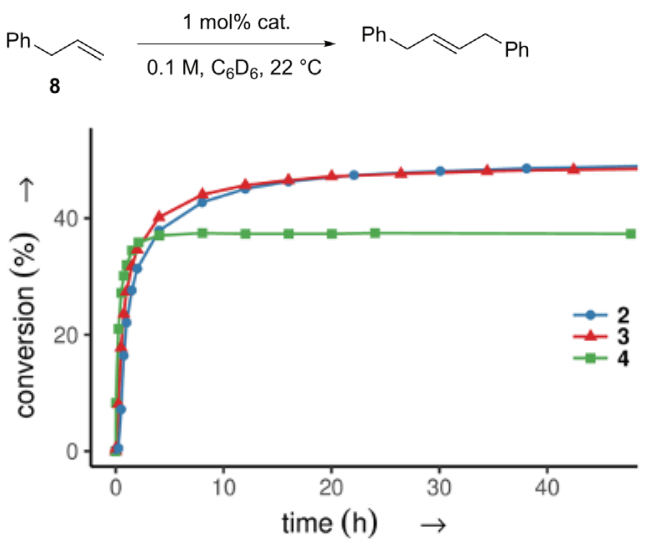

Figure 3: Performance of catalysts 1-4 in self-metathesis of allyl benzene.

\section{Cross-metathesis}

Following a standard system of catalyst characterization, ${ }^{[51]}$ the cross-metathesis process between allyl benzene and cis-1,4-diacetoxybutene was subsequently investigated. The carbene substituent patterns displayed in this case significant differences on the outcome. The bulkiest catalyst 1 yielded 1\% of cross-metathesis product after 1 week, while about $90 \%$ of the catalyst remained unchanged in the mixture (Figure S6). In contrast, the least sterically hindered complex 4 underwent complete decomposition after 2 hours, yielding only $2.5 \%$ of the cross-metathesis product. The carbene substituent patterns in catalysts $\mathbf{2}$ and $\mathbf{3}$, however, resulted in a proper balance between activity and stability leading to good conversions in shorter time (Figure S9). Interestingly, all catalysts exhibited lower E/Z-selectivity in the cross-metathesis process than non-CAAC catalysts GI-Bn, GII, HGI, and HGII (Figure S8). ${ }^{[51]}$

\section{Functional group tolerance}

Since high activity of CAAC catalysts can be attributed to the stability of the alkylidene intermediates, the robustness is an important and valuable property. The integrity of the catalysts was therefore evaluated with respect to time and different additives. Complexes 1-3 exhibited excellent stability at room temperature, being active in metathesis processes from several days up to weeks. In order to evaluate the difference between NHC and CAAC catalysts, catalysts 1-4 were also compared to HGII in self-metathesis of allyl benzene at 
room temperature. Surprisingly, catalyst HGII appeared to be considerably less active at room temperature than the CAAC catalysts. The NHC-catalyst was thus almost completely decomposed after $1 \mathrm{~h}$, producing only $11 \%$ conversion after $20 \mathrm{~h}(10 \%$ within $1 \mathrm{~h}$; also $1-2 \%$ of $\beta$-methylstyrene was formed; Figure S9). In contrast, the least stable CAAC catalyst 4 resulted in $37 \%$ conversion in $4 \mathrm{~h}$ under the same conditions.

Typically, olefin metathesis is performed in non-polar solvents, such as toluene and dichloromethane. However, the solubility of polar substrates, especially natural products and drug precursors, sometimes requires a more polar environment, like methanol or water. Nonfunctionalized Hoveyda-Grubbs $2^{\text {nd }}$ generation catalysts are usually sparingly soluble in methanol and their instability in protic media is an important drawback. \{Nelson, 2014 \#37;Binder, 2007 \#3 3 CAAC-based catalysts can in this case display improved performance, where, even at $0.25 \mathrm{~mol} \%$ loading, catalyst 3 converted $25 \%$ allyl benzene within two weeks (20\% after $34 \mathrm{~h}$ ) in $\mathrm{CD}_{3} \mathrm{OD}$ in a self-metathesis process (Figure S7). This indicates improved tolerance of CAAC catalysts to protic solvents, recently applied in aqueous olefin metathesis. ${ }^{[52]}$

In addition to alcohols, several other classes of compounds are known to be detrimental to olefin metathesis catalysts. ${ }^{[41,53,54]}$ An additive screening protocol, in which the functional group was intentionally decoupled from the substrate, was therefore adopted to explore the functional group tolerance of catalyst 2 in cross- and ring-closing metathesis protocols (Table 2). To distinguish the influence of additives, which did not affect the rate of the fast RCM reaction, they were evaluated in the slower allyl benzene self-metathesis process. Certain aliphatic- and heteroaromatic amines retarded the reactions, whereas other polar functional groups, including anilines and pyrroles, were well tolerated. The results are also indicative of a correlation between the coordinating ability of the additives and their ability to poison the ruthenium catalyst. Strongly coordinating ligands, such as pyridine and imidazole, can in principle bind to the metal center and impair the catalytic cycle, as reflected in the present results. Previous studies have also shown that CAAC complexes bearing pyridine moieties suffer from instability and display poor catalytic activity. ${ }^{[12]}$ 
Table 2: Influence of various nucleophilic additives on cross-metathesis and ring-closing metathesis.

\begin{tabular}{|c|c|c|c|c|c|c|c|}
\hline \multirow[t]{2}{*}{ Additive } & \multicolumn{2}{|c|}{ Conversion (\%) } & \multirow[t]{2}{*}{ Catalyst alive ( 2 h) } & \multirow[t]{2}{*}{ Additive } & \multicolumn{2}{|c|}{ Conversion (\%) } & \multirow[t]{2}{*}{ Catalyst alive (2 h) } \\
\hline & $2 \mathrm{~h}$ & $16 \mathrm{~h}$ & & & $2 \mathrm{~h}$ & $3 \mathrm{~d}$ & \\
\hline- & 23 & 45 & $\checkmark$ & - & 87 & 100 & $\checkmark$ \\
\hline & 19 & 40 & $\checkmark$ & $\sim \mathrm{NH}_{2}$ & 0 & 0 & $x$ \\
\hline & 2 & 19 & $\checkmark$ & & 0 & $<1$ & $\checkmark$ \\
\hline & 1 & 8 & $\checkmark$ & & $>1$ & 4 & $\checkmark$ \\
\hline & 18 & 42 & $\checkmark$ & & 0 & 0 & $\checkmark$ \\
\hline & 22 & 44 & $\checkmark$ & 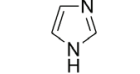 & 0 & 0 & $\checkmark$ \\
\hline & 13 & 40 & $\checkmark$ & & & & \\
\hline & 19 & 41 & $\checkmark$ & & & & \\
\hline & 0 & 2 & $\checkmark$ & & & & \\
\hline
\end{tabular}

\section{Dynamic systems equilibration}

The potential of adopting the CAAC-based catalysts for generation of dynamic covalent systems was furthermore investigated. Complex 2 was in this case selected due to its good balance between activity and stability, enabling sustained catalysis in the equilibrating pool of compounds. In order to investigate the ability of the CAAC-based catalyst to establish thermodynamic equilibria, a dual entry-point analysis of a system containing cis- and trans1,4-diacetoxy-2-butene (9) was performed. [55] Starting from any of the isomers, the E/Z-ratio eventually reached 93:7 (Figure 4 a). In addition, the model cross-metathesis reaction of a 1:1 mixture of allyl benzene and compound Z-9 resulted in equilibrium formation, which upon addition of another equivalent of compound Z-9 reached the same composition as if starting from a 1:2 mixture of the compounds (Figure $\underline{4 b}$ ). 
a)

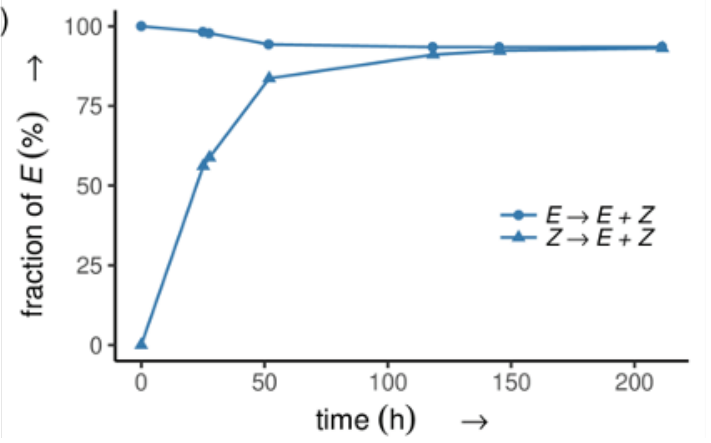

b)

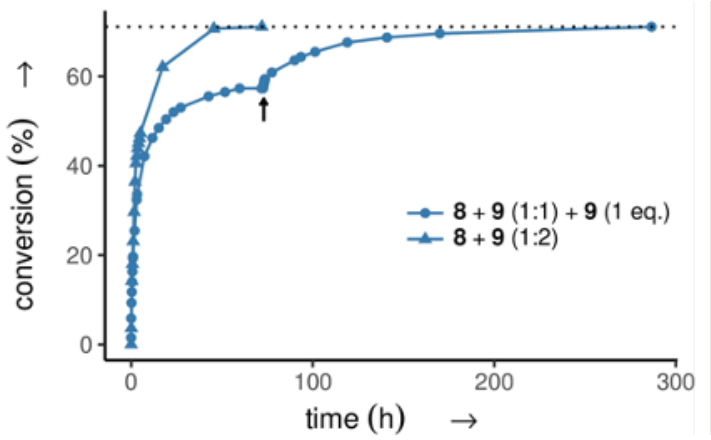

Figure 4: a) Equilibration of system containing either compound E-9 or Z-9; conditions: catalyst 2 (2.5 mol\%), $0.2 \mathrm{M}, \mathrm{C}_{6} D_{6}, 40{ }^{\circ} \mathrm{C}$; b) equilibration of systems containing compounds 8 and $9 ; 1: 1$ mixture reaches the same composition as the 1:2 mixture after addition of another equivalent of compound 9; conditions: catalyst 2 (1 $\mathrm{mol} \%$ ), $0.2 \mathrm{M}, \mathrm{CDCl}_{3}, 22{ }^{\circ} \mathrm{C}$.

These results indicate that CAAC-based catalysts, such as complex $\mathbf{2}$, are well capable of generating prototype dynamic systems under mild conditions at room temperature. The prolonged catalyst lifetime of these structures, in comparison with NHC-based analogs, enabled the establishment of alkene equilibria in cross-metathesis protocols. This combination of catalytic activity and structural integrity is an attractive feature of these catalysts, essential for applications in dynamic chemistry.

\section{CONCLUSIONS}

In conclusion, we have investigated the catalytic performance of several CAAC-based ruthenium complexes in benchmark olefin metathesis reactions under mild conditions. The catalysts were applied to ring-closing-, self-, and cross-metathesis protocols, and found exceptionally stable for weeks in reactions performed at room temperature. The substitution pattern of the aryl substituent of the CAAC moiety was crucial for the catalyst activity, where both highly substituted and sterically unhindered ligands resulted in less performant catalysts. Instead, a delicate balance in the bulkiness of the substituents was necessary, similar to the ethenolysis reaction. ${ }^{[9]}$ In contrast to previous results, ${ }^{[12]}$ the catalysts were found capable of forming tetrasubstituted olefins, albeit at somewhat elevated temperatures. The CAAC complexes showed considerably better stability compared to their NHC analogs, which is especially important at room temperature when catalyst decomposition becomes faster than productive metathesis. From an additive screening protocol, the catalysts were also found stable in the presence of different polar groups, including anilines and pyrroles. These long catalyst lifetimes, together with an increased group tolerance, provide an opportunity for using these catalysts in dynamic covalent chemistry. 


\section{ACKNOWLEDGEMENTS}

This study was in part supported by the Swedish Research Council.

\section{REFERENCES}

[1] V. Lavallo, Y. Canac, C. Präsang, B. Donnadieu, G. Bertrand, Angew. Chem. Int. Ed. 2005, 44, 5705-5709.

[2] V. Lavallo, J. Mafhouz, Y. Canac, B. Donnadieu, W. W. Schoeller, G. Bertrand, J. Am. Chem. Soc. 2004, 126, 8670-8671.

[3] S. Kundu, S. Sinhababu, V. Chandrasekhar, H. W. Roesky, Chem. Sci. 2019, 10, 4727-4741.

[4] M. Melaimi, R. Jazzar, M. Soleilhavoup, G. Bertrand, Angew. Chem. Int. Ed. 2017, 56, 10046-10068.

[5] M. Soleilhavoup, G. Bertrand, Acc. Chem. Res. 2015, 48, 256-266.

[6] M. N. Hopkinson, C. Richter, M. Schedler, F. Glorius, Nature 2014, 510, 485.

[7] A. Kajetanowicz, M. Chwalba, A. Gawin, A. Tracz, K. Grela, Eur. J. Lipid Sci. Technol. 2020, 122, 1900263.

[8] D. L. Nascimento, A. Gawin, R. Gawin, P. A. Guńka, J. Zachara, K. Skowerski, D. E. Fogg, J. Am. Chem. Soc. 2019, 141, 10626-10631.

[9] V. M. Marx, A. H. Sullivan, M. Melaimi, S. C. Virgil, B. K. Keitz, D. S. Weinberger, G. Bertrand, R. H. Grubbs, Angew. Chem. Int. Ed. 2015, 54, 1919-1923.

[10] D. R. Anderson, T. Ung, G. Mkrtumyan, G. Bertrand, R. H. Grubbs, Y. Schrodi, Organometallics 2008, 27, $563-566$.

[11] O. M. Ogba, N. C. Warner, D. J. O’Leary, R. H. Grubbs, Chem. Soc. Rev. 2018, 47, 4510-4544.

[12] D. R. Anderson, V. Lavallo, D. J. O'Leary, G. Bertrand, R. H. Grubbs, Angew. Chem. Int. Ed. 2007, 46, $7262-7265$.

[13] D. L. Nascimento, D. E. Fogg, J. Am. Chem. Soc. 2019, 141, 19236-19240.

[14] R. Gawin, A. Kozakiewicz, P. A. Guńka, Dąbrowski Paweł, K. Skowerski, Angew. Chem. Int. Ed. 2017, 56, 981-986.

[15] Gawin Rafał, A. Tracz, Chwalba Michał, A. Kozakiewicz, B. Trzaskowski, K. Skowerski, ACS Catal. 2017, 7, 5443-5449.

[16] Y. Zhang, Y. Qi, S. Ulrich, M. Barboiu, O. Ramström, Mater. Chem. Front. 2020, 4, 489-506.

[17] P. Frei, R. Hevey, B. Ernst, Chem. Eur. J. 2019, 25, 60-73.

[18] A. M. Hartman, R. M. Gierse, A. K. H. Hirsch, Eur. J. Org. Chem. 2019, 2019, 3581-3590.

[19] A. G. Orrillo, A. M. Escalante, M. Martinez-Amezaga, I. Cabezudo, R. L. E. Furlan, Chem. Eur. J. 2019, $25,1118-1127$.

[20] Y. Zhang, Y. Zhang, O. Ramström, Catal. Rev. 2019, 62, 66-95.

[21] O. Š. Miljanić, Chem 2017, 2, 502-524.

[22] Y. Zhang, M. Barboiu, Chem. Rev. 2016, 116, 809-34.

[23] I. Azcune, I. Odriozola, Eur. Polym. J. 2016, 84, 147-160.

[24] R.-C. Brachvogel, M. von Delius, Eur. J. Org. Chem. 2016, 2016, 3662-3670.

[25] J.-M. Lehn, Angew. Chem. Int. Ed. 2015, 54, 3276-3289.

[26] A. Herrmann, Chem. Soc. Rev. 2014, 43, 1899-1933.

[27] L. Hu, F. Schaufelberger, B. J. J. Timmer, M. A. Flos, O. Ramström, in Kirk-Othmer Encycl. Chem. Technol., 2014, pp. 1-25.

[28] M. C. Misuraca, E. Moulin, Y. Ruff, N. Giuseppone, New J. Chem. 2014, 38, 3336-3349.

[29] A. Wilson, G. Gasparini, S. Matile, Chem. Soc. Rev. 2014, 43, 1948-62.

[30] C. A. Denard, H. Huang, M. J. Bartlett, L. Lu, Y. Tan, H. Zhao, J. F. Hartwig, Angew. Chem. Int. Ed. 2014, $53,465-469$.

[31] L. Hunter, G. C. Condie, M. M. Harding, Tetrahedron Lett. 2010, 51, 5064-5067.

[32] S.-A. Poulsen, L. F. Bornaghi, Bioorg. Med. Chem. 2006, 14, 3275-3284.

[33] P. C. M. van Gerven, J. A. A. W. Elemans, J. W. Gerritsen, S. Speller, R. J. M. Nolte, A. E. Rowan, Chem. 
Commun. 2005, 3535-3537.

[34] K. C. Nicolaou, R. Hughes, S. Y. Cho, N. Winssinger, H. Labischinski, R. Endermann, Chem. Eur. J. 2001, 7, 3824-3843.

[35] K. C. Nicolaou, R. Hughes, S. Y. Cho, N. Winssinger, C. Smethurst, H. Labischinski, R. Endermann, Angew. Chem. Int. Ed. 2000, 39, 3823-3828.

[36] C. Brändli, T. R. Ward, Helv. Chim. Acta 1998, 81, 1616-1621.

[37] T. Giger, M. Wigger, S. Audetat, S. A. Benner, Synlett 1998, 688-691.

[38] B. J. Ireland, B. T. Dobigny, D. E. Fogg, ACS Catal. 2015, 5, 4690-4698.

[39] S. H. Hong, A. G. Wenzel, T. T. Salguero, M. W. Day, R. H. Grubbs, J. Am. Chem. Soc. 2007, 129, 7961-7968.

[40] S. Manzini, A. Poater, D. J. Nelson, L. Cavallo, A. M. Z. Slawin, S. P. Nolan, Angew. Chem. Int. Ed. 2014, 53, 8995-8999.

[41] D. J. Nelson, S. Manzini, C. A. Urbina-Blanco, S. P. Nolan, Chem. Commun. 2014, 50, 10355-10375.

[42] D. Butilkov, A. Frenklah, I. Rozenberg, S. Kozuch, N. G. Lemcoff, ACS Catal. 2017, 7, 7634-7637.

[43] K. Skowerski, G. Szczepaniak, C. Wierzbicka, L. Gulajski, M. Bieniek, K. Grela, Catal. Sci. Technol. 2012, 2, 2424-2427.

[44] A. E. Samkian, Y. Xu, S. C. Virgil, K.-Y. Yoon, R. H. Grubbs, Organometallics 2020, 39, 495-499.

[45] J. Zhang, S. Song, X. Wang, J. Jiao, M. Shi, Chem. Commun. 2013, 49, 9491-9493.

[46] J. P. Jordan, R. H. Grubbs, Angew. Chem. Int. Ed. 2007, 46, 5152-5155.

[47] S. H. Hong, R. H. Grubbs, J. Am. Chem. Soc. 2006, 128, 3508-3509.

[48] Y. A. Lin, B. G. Davis, Beilstein J. Org. Chem. 2010, 6, 1219-1228.

[49] A. H. Hoveyda, P. J. Lombardi, R. V. O’Brien, A. R. Zhugralin, J. Am. Chem. Soc. 2009, 131, 8378-8379.

[50] T. R. Hoye, H. Zhao, Org. Lett. 1999, 1, 1123-1125.

[51] T. Ritter, A. Hejl, A. G. Wenzel, T. W. Funk, R. H. Grubbs, Organometallics 2006, 25, 5740-5745.

[52] M. Nagyházi, G. Turczel, Á. Balla, G. Szálas, I. Tóth, G. T. Gál, B. Petra, P. T. Anastas, R. Tuba, ChemCatChem 2020, 12, 1953-1957.

[53] P. Compain, Adv. Synth. Catal. 2007, 349, 1829-1846.

[54] Z. J. Wang, W. R. Jackson, A. J. Robinson, Green Chem. 2015, 17, 3407-3414.

[55] F. Schaufelberger, B. J. J. Timmer, O. Ramström, in Dynamic Covalent Chemistry. Principles, Reactions, and Applications, 2018, pp. 1-30. 